\title{
EFECTIVIDAD DEL FENBENDAZOL Y PRAZIQUANTEL PARA EL CONTROL EN DOSIS ÚNICA DE NEMÁTODES Y CÉSTODES EN PERROS
}

\author{
Manuel Cárdenas R. ${ }^{1}$, Amanda Chávez V. ${ }^{2,3}$ y Eva Casas A. ${ }^{2}$
}

\section{Abstract}

The objective of the study was to evaluate the therapeutic effectiveness of the fenbendazole-praziquantel combination against nematode and cestode parasitism in dogs. A single dose of $100 \mathrm{mg}$ of fenbendazole and $5 \mathrm{mg}$ of praziquantel per kilogram of body weight was orally administered. Ten female/male 12-14 weeks old pupies were naturally infected with Toxocara canis and Dipylidium caninum, and divided in a control and treated groups. Adult forms of the parasites were searched in the necropsy at day 4 after treatment. The effectiveness was 92.5 and $100 \%$ against $T$. canis and $D$. caninum, respectively, and without side effects in treated animals. The results showed that the fenbendazole-praziquantel combination at the specified dose was highly effective against these parasites.

Key words: effectiveness, fenbedazole, praziquantel, T. canis, D. caninum

\section{RESUMEN}

El objetivo del estudio fue evaluar la efectividad terapéutica en dosis única, de la combinación fenbendazol-praziquantel contra nemátodes y céstodes en perros. Se emplearon 10 cachorros machos y hembras, de 12 a 14 semanas de edad, infectados naturalmente con Toxocara canis y Dipylidium caninum, divididos en dos grupos: control no tratado y tratado. Los fármacos se administraron por via oral en cantidades de $100 \mathrm{mg}$ de fenbendazol y $5 \mathrm{mg}$ de praziquantel por kilogramo de peso vivo. En la necropsia realizada al $4^{\circ}$ día post tratamiento se encontró una efectividad del 92.5 y $100 \%$ contra $T$. canis y D. caninum, respectivamente, y sin efectos secundarios para el animal. Este resultado demuestra que la combinación fenbendazolpraziquantel a la dosis descrita, es altamente efectiva contra los parásitos en mención y segura para el animal tratado.

Palabras clave : efectividad, fenbendazol, praziquantel, T. canis, D. caninum

\author{
${ }^{1}$ Práctica privada \\ ${ }^{2}$ Laboratorio de Microbiología y Parasitología Veterinaria, FMV-UNMSM \\ ${ }^{3}$ E-mail: a_chavez_g@hotmail.com
}




\section{INTRODUCCIÓN}

El Médico Veterinario especialista en pequeñas especies está comprometido en cumplir con las tareas dirigidas a la prevención, diagnóstico y tratamiento de las enfermedades parasitarias en perros y gatos, algunas de las cuales son zoonóticas (Acha y Szyfres, 1986; Botero y Restrepo, 1998). En este sentido, la industria farmacológica veterinaria ha desarrollado varias drogas para el tratamiento antiparasitario, invirtiendo tiempo y dinero para lograr combinaciones idóneas, así como para determinar las dosis más apropiadas.

Las parasitosis intestinales que afectan a los caninos tienen como causales a nemátodes como el Toxocara canis, Toxascaris leonina, Ancylostoma caninum y Trichuris vulpis, y céstodes como Dipylidium caninum, Taenia hidatigena, Taenia pisiformis y Echinococcus granulosus. Estos pueden producir trastornos clínicos tales como disminución del apetito, mal aprovechamiento de los alimentos por trastornos en el proceso de la digestión y absorción, así como anemia, hipoproteinemia, retraso en el crecimiento, disminución de peso $\mathrm{y}$, en algunos casos, mortalidad (Diez et al., 1999; Sánchez et al., 1999).

T. canis y D. caninum son los parásitos de mayor frecuencia en caninos en el país. Estos parásitos pueden afectar ocasionalmente al hombre, especialmente a niños, constituyendo zoonosis parasitarias de amplia morbilidad, aunque escasamente definidas (García et al., 2002).

Debido a la presentación mixta de estas parasitosis, se requiere combinar diversos antiparasitarios para lograr alternativas que sean efectivas para su control. Varios principios activos han sido empleados en el tratamiento de nemátodes y céstodes, con diversos grados de eficacia (Ulloa, 1995). Se dispone de tratamientos que emplean las combinaciones de levamisol y niclosamida, piperazina y praziquantel, pamoato de pirantel y praziquantel. El fenbendazol y praziquantel ha sido administrado durante tres días consecutivos en ayunas o con los alimentos en cachorros desde las 2 semanas de edad, en perras gestantes entre los días 42 y 50 , ó en lactación entre los días 12 y 18 después del parto, sin que se presenten efectos adversos $y$ con un alto porcentaje de efectividad (Booth y McDonald, 1987).

El objetivo del presente estudio fue determinar la efectividad antinematódica y anticestódica de la combinación de fenbendazol y praziquantel, administrada en dosis única en caninos.

\section{Materiales y Métodos}

\section{Lugar experimental y animales}

La evaluación de la efectividad del antiparasitario fue realizada en caniles individuales del Laboratorio de Parasitología de la Facultad de Medicina Veterinaria de la Universidad Nacional Mayor de San Marcos. Se utilizaron 10 cachorros, entre machos y hembras, cuyas edades fluctuaron entre 12 a 14 semanas y que fueron naturalmente parasitados con $T$. canis y D. caninum. Se dividió a los animales en dos grupos de 5 animales cada uno: grupo Control y grupo Tratado. Se les dio alimento balanceado para cachorros 3 veces por día y agua ab libitum; asimismo, el piso de cada canil se cubrió con papel para facilitar la recolección de las heces y posterior observación de la presencia de parásitos.

\section{Fármacos}

Se utilizaron comprimidos comerciales que contenían $500 \mathrm{mg}$ de fenbendazole y 25 mg de praziquantel. La dosis empleada en el grupo tratado fue de $100 \mathrm{mg}$ de fenbendazole y $5 \mathrm{mg}$ de praziquantel por kilogramo de peso vivo. 


\section{Identificación de los parásitos}

Los animales fueron sacrificados entre 72 y 96 horas posteriores al tratamiento. Los intestinos fueron seccionados e incididos longitudinalmente para realizar la extracción de los parásitos que se pudiesen encontrar en la mucosa intestinal. Los parásitos fueron colocados en placas petri con suero fisiológico para su identificación y cuantificación. La mucosa de los intestinos se raspó para realizar el recuento directo de los escólices de las tenias.

\section{Efectividad de la medicación}

Los resultados fueron expresados en porcentajes de efectividad para la droga en evaluación, empleando la siguiente fórmula:

$$
\begin{aligned}
& \text { Parásitos }^{1} \\
& \text { del Control }
\end{aligned}-\begin{aligned}
& \text { Parásitos } \\
& \text { del Tratado }
\end{aligned}
$$

Efectividad $=\longrightarrow$ x100

Parásitos

del Control

donde 1 se refiere al número promedio de parásitos en cada grupo experimental.

Los resultados de efectividad se agruparon de la siguiente forma (Kassai, 1998), donde se considera hasta moderadamente efectivo como un grado aceptable de efectividad:

- Muy efectivo (superior al 98\%)

- Efectivo (90 a 98\%)

- Moderadamente efectivo (80 a 89\%)

- Insuficientemente efectivo $(<80 \%)$

\section{Resultados y Discusión}

En el tratamiento antiparasitario se requiere emplear antihelmínticos de eficacia comprobada. Es además de suma importancia definir el momento adecuado para la desparasitación, ya que un mismo tratamiento puede ser correcto y estar bien justificado en un momento dado, mientras que en otro puede ser inadecuado e inútil. En la práctica, el valor de un antihelmíntico depende de factores tales como el nivel del espectro, efecto residual, actividad frente a estadios adultos, juveniles y larvarios de los vermes, vía de aplicación, seguridad y costo (Kassai, 1998).

En el presente estudio se midió la efectividad de la combinación del fenbendazole y praziquantel en dosis única para el tratamiento de Toxocara canis y Dipylidium caninum. Las cargas parasitarias en los 5 cachorros del grupo control variaron de 9 a 31 especímenes de $T$. canis y de 5 a $28 D$. caninum; mientras que en cachorros del grupo tratado, se encontró especímenes de $T$. canis en dos de ellos. El número de parásitos encontrados en la necropsia para los grupos experimentales se muestra en el Cuadro 1. La ausencia de D. caninum en heces despues del tratamiento pudo deberse a la acción directa del praziquantel sobre el tegumento del parásito (Goodman y Gilman, 1996).

Los resultados indican que la combinación de fenbendazol y praziquantel, administrado en dosis única, fue efectiva para $T$. canis y muy efectiva para D. caninum. Estos resultados concuerdan con otros obtenidos al evaluar las drogas en forma individual; así, en un estudio se demostró que la eficacia del fenbendazol y praziquantel fue de 95 y 98\%, respectivamente (Roudebush, 1985). En otro estudio utilizando fenbendazol contra Ascaris sp. durante 3 días consecutivos en dosis de $50 \mathrm{mg} / \mathrm{kg}$ de p.v. se obtuvo una eficacia del 98 al 100\% (Stepehen, 1994), en tanto que en otros dos estudios con fenbendazol en dosis única de $100 \mathrm{mg} / \mathrm{kg}$ de p.v. se observó una reducción del 99 y 100\%, respectivamente, de huevos de Toxocara sp. en heces (Fisher et al., 1993).

Las evaluaciones de antiparasitarios han mostrado resultados diversos en términos de efectividad. Se tienen reportes favorables al uso de praziquantel contra 
Cuadro 1. Porcentaje de efectividad de la combinación de fenbendazole y praziquantel contra Toxocara canis y Dipylidium caninum (Lima, 2004)

\begin{tabular}{cccccc}
\hline \multirow{2}{*}{ Grupo } & \multicolumn{2}{c}{$\begin{array}{c}\text { Promedio de parásitos } \\
\text { en el intestino delgado }\end{array}$} & & \multicolumn{2}{c}{ Efectividad (\%) } \\
\cline { 2 - 3 } \cline { 5 - 6 } \cline { 5 - 6 } T. canis & D. caninum & & T. canis & D. caninum \\
\hline Control & 16.6 & 15.6 & & - & - \\
Tratado & 0.8 & 0 & & 95.2 & 100.0 \\
\hline
\end{tabular}

Dipylidium caninum, Taenia sp. y Echinococcus granulosus en dosis de $5 \mathrm{mg} /$ kg de p.v. (Leguía, 2002). Por otro lado, los estudios realizados por Catton y van Schalkwyk (2004) presentaron eficacias de $39 \%$ contra ancilostomas y $49 \%$ contra ascáridos, toda vez que se usó una dosis de $50 \mathrm{mg}$ de fenbendazol y $2.5 \mathrm{mg}$ de praziquantel por kilogramo de peso vivo; es decir, la mitad de la dosis que se recomienda para estos parásitos.

Para el estudio de la efectividad de drogas antiparasitarias se recomienda el sacrificio de los animales tratados para encontrar el número exacto de parásitos no afectados; no obstante, estas evaluaciones son bastante complicadas. Por otro lado, las técnicas basadas en los recuentos fecales no son exactas debido a factores tales como la intermitencia de la eliminación de huevos, parásitos productores de bajo número de huevos, mayor presencia de parásitos machos, muestras pequeñas o mal conservadas y errores en la ejecución de la técnica (Kassai, 1998).

En el presente estudio, se observó que los animales tratados eliminaban nemátodes muertos entremezclados con las heces hasta el día anterior a la eutanasia; además, se observó mejora en el aspecto y consistencia de las heces en el grupo tratado, así como una mejora en el ánimo y el apetito de los cachorros del grupo tratado en comparación con los del grupo control.
Los animales no manifestaron síntomas adversos luego de la administración de la combinación de fenbendazol y praziquantel indicando una buena tolerancia en las dosis utilizadas; sin embargo, Atías (1995) señala que la administración de praziquantel vía oral puede ocasionalmente causar dolor abdominal, fiebre, cefalea, vértigo y somnolencia.

En el examen anatomopatológico se observó que el grupo control presentaba el tracto intestinal brilloso y engrosado, con zonas congestionadas y ligeramente hemorrágicas, principalmente en la zona duodenal, tercio anterior y posterior del yeyuno; encontrándose en las dos primeras zonas mencionadas el mayor porcentaje de T. canis (parásitos redondos, blanquecinos, de aproximadamente $10 \mathrm{~cm}$. de longitud), mientras que en la zona del tercio posterior del yeyuno se encontraron los $D$. caninum (parásitos alargados, con numerosos segmentos, planos y blanquecinos de aproximadamente $20 \mathrm{~cm}$ de longitud). Estos se encontraban prendidos con sus escólices, llegando el extremo posterior hasta la zona proximal del íleon (Fig. 1a). La mucosa se encontró engrosada con una coloración amarillenta y con un exudado mucoso abundante, compatible con una «enteritis catarral». En el grupo tratado, el tracto intestinal estaba aparentemente normal, presentando un color rosado con ligero enrojecimiento en el tercio distal del yeyuno (Fig. 1b). 

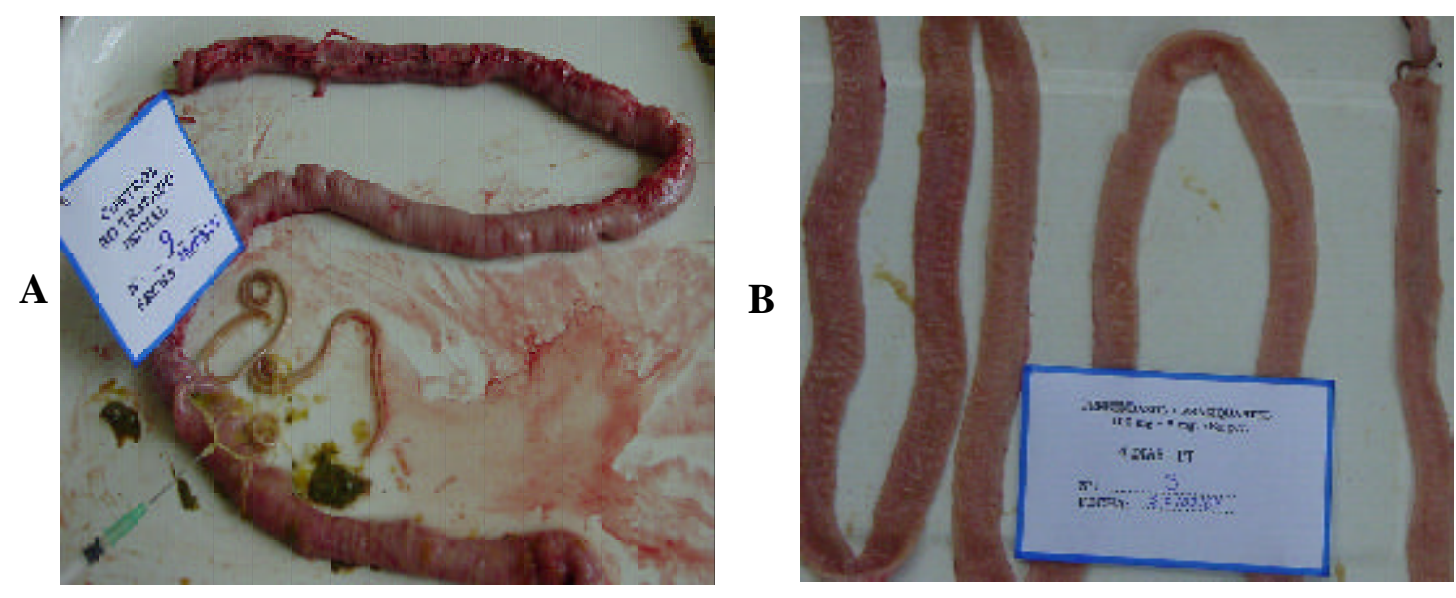

Figura 1. Intestino delgado de un cachorro del grupo control (A) con presencia de $T$. canis y $D$. caninum, y de un cachorro del grupo tratado (B) sin presencia de parásitos y con mucosa normal

\section{CONCLuSiones}

- La combinación de fenbendazol y praziquantel administrado vía oral, en dosis única, a razón de $100 \mathrm{mg} / \mathrm{kg}$ de p.v. de fenbendazol y $5 \mathrm{mg} / \mathrm{kg}$ de p.v. de praziquantel es efectiva contra Toxocara canis y muy efectiva contra Dipilidium caninum.

- La combinación empleada puede ser utilizada sin esperarse efectos colaterales o reacciones secundarias adversas.

\section{Limeratura Citada}

1. Acha, P.; B. Szyfres. 1986. Zoonosis y enfermedades transmisibles comunes al hombre y a los animales. $2^{\mathrm{a}}$ ed. $\mathrm{p}$ 844850. Organización Panamericana de la Salud. Washington.

2. Atias, A. 1995. Parasitología clínica. $3^{\text {a }}$ ed. p 109-110. Ed. Mediterráneo. Santiago de Chile.

3. Booth, N.; L. Mc Donald. 1987. Farmacología y terapéutica veterinaria. p 157-200. Ed. Acribia. España.
4. Botero, D.; M. Restrepo. 1998. Parasitosis humana. Corporación para investigaciones biológicas. $3^{\text {a }}$ ed. p 335340. Medellín, Colombia.

5. Catton, D.; P. van Schalkwyk. 2004. Eficacia de cuatro antihelmínticos contra ascaridos y ancylostomas en perros infestados naturalmente. Mundo Veterinario 7: 61-62.

6. Diez, P.; N. Diez; M. Morrondo. 1999. Nematodiosis: toxocarosis, toxascariosis, ancilostomatidosis, tricuriosis, estrongiloidosis, espirocercosis y olulanosis. En: Parasitología veterinaria. M. Cordero del Campillo; F. Rojo (eds). p 626-635. Ed. McGraw-Hill-Interamericana. Madrid.

7. Fisher, M.; D. Jacobs; M. Hutchinson; E. Abbott. 1993. Efficacy of fenbendazole and piperazine against developing stages of Toxocara and Toxascaris in dogs. Vet. Rec. 132: 473475.

8. García, M.; A. Chávez; E. Casas; D. Díaz; J. Avendaño; B. Campos; F. Loayza. 2002. Estudio de las zoonosis parasitarias de localización ocular en el instituto de oftalmología (INO) durante el periodo 1985-1999. Rev. Inv. Vet., Perú. 13: 78-83. 
9. Goodman, A.; F. Gilman. 1996. Las bases farmacológicas de la terapéutica. $9^{a}$ ed. p 1234. Ed. McGraw-Hill Interamericana. México DF.

10. Kassai, T. 1998. Helmintología veterinaria. p 68-88. Ed. Acribia. Zaragoza.

11. Leguía, G. 2002. Enfermedades parasitarias de perros y gatos. $2^{\mathrm{a}}$ ed. p 155. Ed. Del Mar. Lima.

12. Roudebush, P. 1985. An updated guide to the chemotheraphy of small animal intestinal parasites. Canine Pract. 12: 7-20.

13. Sánchez, C.; J. Quílez; E. Del Cacho. 1999. Cestodiosis: teniosis, equinoco- cosis, dipilidiosis, mesocestoidosis $\mathrm{y}$ difilobotriosis. En: Parasitología veterinaria. M. Cordero del Campillo; F. Rojo (eds). p 636-647. Ed. McGraw-HillInteramericana. Madrid.

14. Stepehen, C. 1994. Efficacy of fenbendazole aganist in dogs. Vet. Rec. 1: 988-990.

15. Ulloa, I. 1995. Evaluación del nitroscanato contra tenias y nemátodos en caninos. Tesis de Médico Veterinario. Facultad de Medicina Veterinaria, Univ. Nacional Mayor de San Marcos, Lima. 29 p. 